\title{
Force spectroscopy using bimodal frequency modulation atomic force microscopy
}

\author{
M. Deniz Aksoy* and A. Atalar ${ }^{\dagger}$ \\ Electrical and Electronics Engineering Department, Bilkent University TR-06800 Bilkent, Ankara, Turkey
}

(Received 22 September 2010; revised manuscript received 13 December 2010; published 15 February 2011)

\begin{abstract}
We propose a force-spectroscopy technique where a higher order mode of a cantilever is excited simultaneously with the first. Resonance tracking of both vibration modes through a frequency modulation scheme provides a way to extract topographical information and the gradient of the tip-sample interaction within a single surface scan. We provide an analytic treatment of the scheme, derive expressions relating frequency shifts of the higher mode and the tip-sample forces, and offer two methods of improving the accuracy of reconstruction of the force gradient. Finally, we confirm our predictions by numerical simulations.
\end{abstract}

DOI: 10.1103/PhysRevB.83.075416

PACS number(s): 68.37.Ps

\section{INTRODUCTION}

Since its invention, atomic force microscopy (AFM) has been used in a vast variety of applications and has proven to be a powerful tool in nanometer science. ${ }^{1}$ Although nanoscale resolution of the surface topography achieved by AFM reveals a lot of information about the sample, it is also desirable to identify and differentiate compositional features. It is possible to perform frequency-versus-distance measurements by tracking frequency changes of a vibrating lever to calculate tip-sample forces. ${ }^{2-4}$ Such spectroscopic measurements suffer from lateral and vertical thermal drift, and imaging speed is severely reduced by the requirement of scanning in the normal direction. ${ }^{5}$ Several techniques have been proposed for simultaneous and faster acquisition of the compositional features and the topographical information. Sahin et al. introduced the use of harmonic cantilevers to recover time resolved forces acting on the tip from harmonics generated by the nonlinear tip-sample interaction. ${ }^{6,7}$ Recently, bimodal amplitude modulation AFM techniques have been developed whereby a higher order flexural mode is excited simultaneously with the first mode to achieve increased phase sensitivity to compositional features at the higher order mode ${ }^{8-10}$ Chawla et al. proposed a hybrid technique, bringing together ideas from both frequency and amplitude modulation AFM techniques, to extract the tip-sample force curve $F_{\mathrm{ts}}(z){ }^{11}$

Slow transient response of the probe with a large quality factor is a fundamental restriction on the available bandwidth in amplitude modulation AFM. ${ }^{12}$ Frequency modulation AFM ${ }^{13}$ seems to circumvent this problem, since the frequency shift of the vibration is almost instantaneous and is independent of the quality factor of the resonator, therefore imaging bandwidth can be chosen arbitrarily large. Recent development of low-noise, wide-bandwidth frequency demodulators open up the possibility of exploiting frequency modulation AFM schemes in different ways. ${ }^{14}$ One possibility is to capture the rapid frequency shifts of a higher mode of a cantilever to extract the properties of the tip-sample forces.

In this paper, we propose a fast force-spectroscopy technique in which two modes of a cantilever, having resonant frequencies $\bar{f}_{1}$ and $\bar{f}_{2}$ (not necessarily an integer multiple of each other) with $\bar{f}_{1} \ll \bar{f}_{2}$, are excited in such a way that the amplitudes of both components of the vibration $\left(A_{1}, A_{2}\right)$ stay constant. As seen in Fig. 1, the modes are operated as independent self-sustained oscillators using two separate positive feedback loops, therefore implementing a bimodal frequency modulation AFM scheme. We show that we can extract the tip-sample force gradient along with the surface topography by recording the instantaneous frequency shifts of both vibration modes for small amplitudes of the higher mode vibration such that $A_{2} \ll A_{1}$. In Sec. II, expressions relating surface forces to the instantaneous frequency shift of the higher mode are presented, and the conditions for which those expressions remain valid are derived. In Sec. III, simple recovery algorithms to find the force gradient from the measured frequency shift are discussed. Theoretical predictions are tested by numerical simulations in Sec. IV.

\section{THEORY}

Referring to Fig. 2, the instantaneous tip-sample distance $z(t)$ is written as

$$
z(t)=z_{1}(t)+A_{2} \cos \left(2 \pi f_{2} t-\phi\right),
$$

with

$$
z_{1}(t)=Z_{0}+A_{1} \cos \left(2 \pi f_{1} t\right),
$$

where $Z_{0}$ is the base-sample (or the average tip-sample) separation, $f_{1}$ and $f_{2}$ are the instantaneous frequencies of the components of the biharmonic vibration centered around $\bar{f}_{1}$ and $\bar{f}_{2}$ with $\phi$ being the phase shift of the higher mode vibration with respect to the time reference of the first mode vibration.

As $Z_{0}$ is decreased, the tip enters into a force field, i.e., the cantilever tip starts spending time at the attractive and repulsive force regimes for increasingly longer intervals within the period $T_{1}=1 / \bar{f}_{1}$. The presence of the force field modulates the instantaneous frequencies of vibration modes. As it is traditionally exploited in single mode frequency modulation AFM experiments, ${ }^{13}$ the frequency shift of the first mode $\left(\Delta f_{1}=f_{1}-\bar{f}_{1}\right)$ can be used as a feedback parameter to extract the topographical variation, whereas the frequency shift of the higher mode $\left(\Delta f_{2}=f_{2}-\bar{f}_{2}\right)$ is sensitive to sample properties such as the density and the elasticity. ${ }^{11}$ However, the exact nature of the relation between $\Delta f_{2}$ and the nonlinear tip-sample interaction is complicated and therefore deserves a careful treatment.

We assume that the motion of the modes of the cantilever can be described by independent weakly disturbed harmonic 


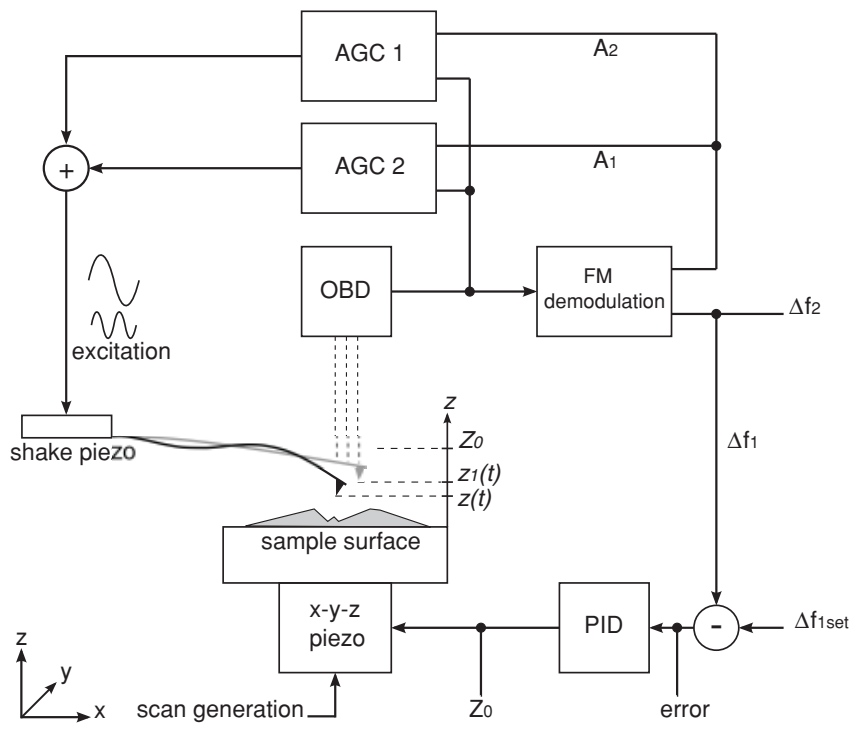

FIG. 1. Schematic description of the proposed technique. Two modes of a cantilever driven simultaneously. $z(t)$ is the instantaneous tip position with respect to the sample, and $z_{1}(t)$ represents the first mode of vibrations. $z(t)$ can be detected using an optical beam deflection (OBD) system. Automatic gain circuitries (AGC) on the positive feedback loop are used to maintain the constancy of the vibration amplitudes, and a single proportional-integral-derivative (PID) control block is used to maintain a fixed base sample separation $\left(Z_{0}\right)$.

oscillators with a spring constant $k$ and an effective mass $m$. The instantaneous frequency shift, $\Delta \widetilde{f}_{2}$, due to nonlinear tip-sample interaction can be calculated by a first-order perturbation theory using the Hamilton-Jacobi approach ${ }^{3}$ as

$$
\begin{aligned}
\Delta \widetilde{f}_{2}(t) \approx & -\frac{\bar{f}_{2}^{2}}{k_{2} A_{2}} \int_{t-\frac{T_{2}}{2}}^{t+\frac{T_{2}}{2}} F_{\mathrm{ts}}\left(z_{1}(\tau)+A_{2} \cos \left(2 \pi \bar{f}_{2} \tau-\phi\right)\right) \\
& \times \cos \left(2 \pi \bar{f}_{2} \tau-\phi\right) d \tau
\end{aligned}
$$

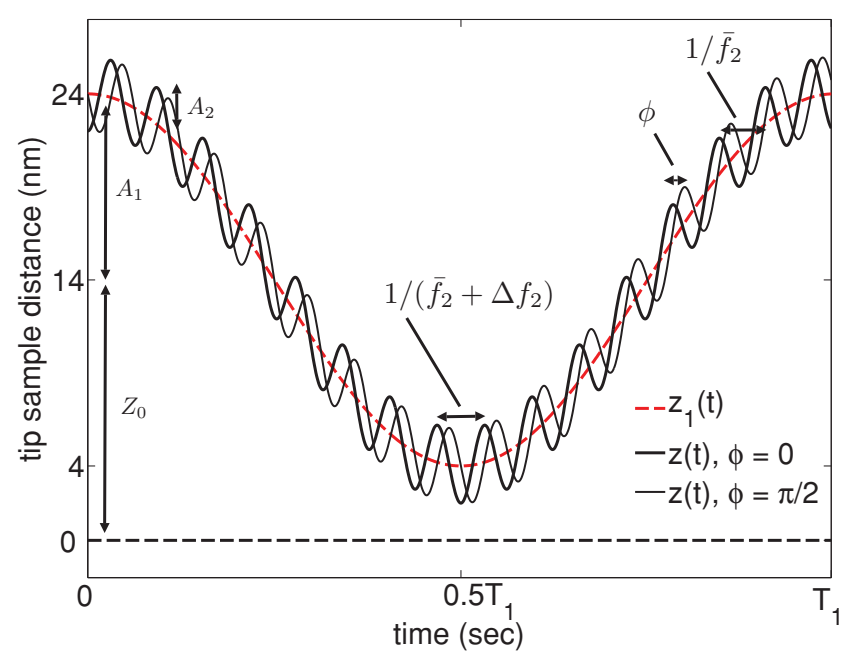

FIG. 2. (Color online) Tip trajectory with respect to time during a single period of the first mode vibration for different values of $\phi$. $\bar{f}_{2} \approx 16 \bar{f}_{1}, Z_{0}=14 \mathrm{~nm}$, while $A_{1}=10$ and $A_{2}=2 \mathrm{~nm}$.

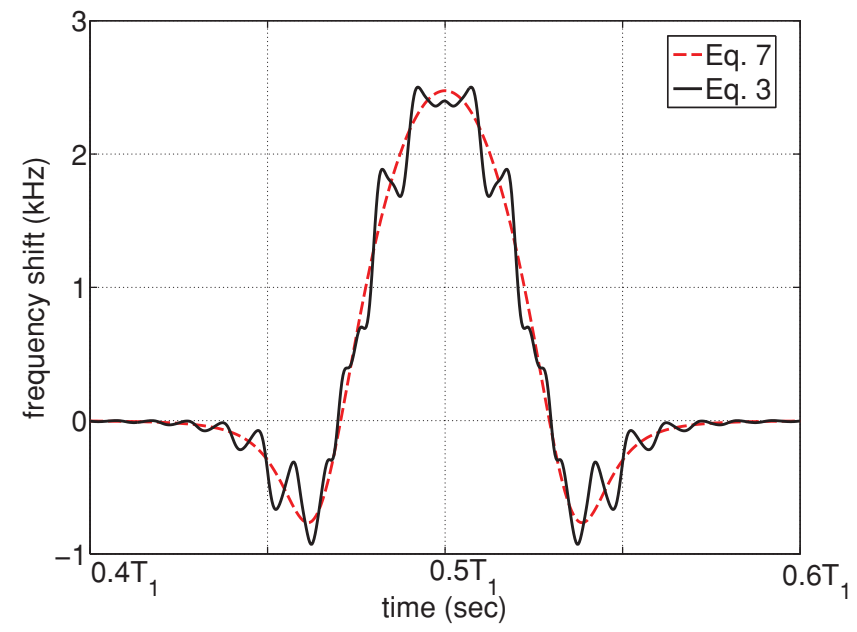

FIG. 3. (Color online) The dashed curve is the instantaneous frequency shift of the higher mode, $\Delta \tilde{f}_{2}(t)$, with respect to time as obtained from Eq. (3) $\left(\bar{f}_{2} \approx 100 \bar{f}_{1}\right)$. The solid curve is the low-pass filtered version of the frequency shift, $\Delta f_{2}(t)$, from Eq. (7). Equivalently, it is the response of frequency demodulator with a bandwidth of $20 \bar{f}_{1} . A_{1}=10 \mathrm{~nm}$, $A_{2}=0.5 \mathrm{~nm}, Z_{0}=10 \mathrm{~nm}, \bar{f}_{1}=100 \mathrm{kHz}, F_{\text {max }}=5 \mathrm{nN}, S_{\text {rep }}=$ $150 \mathrm{nN} \mathrm{nm}^{-2}$ where $F_{\mathrm{ts}}$ is given by Eq. (21).

where $k_{2}$ is the spring constant of the higher mode, $T_{2}=1 / \bar{f}_{2}$ is the period of this mode, and $F_{\mathrm{ts}}(z)$ is the force acting on the tip. Figure 3 shows a plot of instantaneous frequency shift of the higher mode as a function of time as obtained from Eq. (3). This figure reveals that the instantaneous frequency shift has small wiggles with a period of $T_{2}$ and with a phase shift of $\phi$. Although Eq. (3) describes the frequency shift of the higher mode to a great accuracy, this equation does not allow an easy way of determining $F_{\mathrm{ts}}(z)$ from the measured $\Delta \widetilde{f}_{2}(t)$. Moreover, it is possible to measure $\Delta \widetilde{f}_{2}(t)$ only with a wideband demodulator having a bandwidth larger than $\bar{f}_{2}$. If $\bar{f}_{2}$ is a high frequency, it is not feasible to measure the instantaneous frequency $\Delta \widetilde{f}_{2}(t)$ at that high speed with presently available electronic circuits. Therefore we need to obtain an expression that does not contain the high-frequency wiggles at $\bar{f}_{2}$.

Since $T_{2} \ll T_{1}, \tau$ in Eq. (3) lies in the close proximity of $t$, and hence we can write $z_{1}(\tau)$ of Eq. (2) as a Taylor series expansion of the first order

$$
\begin{aligned}
z_{1}(\tau) & \approx z_{1}(t)+\frac{d z_{1}(t)}{d \tau}(\tau-t) \\
& =z_{1}(t)-2 \pi A_{1} f_{1} \sin \left(2 \pi f_{1} t\right)(\tau-t) .
\end{aligned}
$$

Assuming that $f_{1} \approx \bar{f}_{1}$ in Eq. (4) and through a change of variables $\theta=2 \pi \bar{f}_{2}(\tau-t)$ in Eq. (3) we arrive at

$$
\begin{aligned}
\Delta \widetilde{f}_{2}(t) \approx & -\frac{\bar{f}_{2}}{2 \pi k_{2} A_{2}} \int_{-\pi}^{\pi} F_{\mathrm{ts}}\left(z_{1}(t)-A_{1} \frac{\bar{f}_{1}}{\bar{f}_{2}} \theta \sin \left(2 \pi \bar{f}_{1} t\right)\right. \\
& \left.+A_{2} \cos \left(\theta+\phi^{\prime}\right)\right) \cos \left(\theta+\phi^{\prime}\right) d \theta,
\end{aligned}
$$

where $\phi^{\prime}=2 \pi \bar{f}_{2} t-\phi$. Since $\bar{f}_{1} \ll \bar{f}_{2}$, Eq. (5) is a good approximation to Eq. (3). 
If the following condition is satisfied for $-\pi \leqslant \theta \leqslant \pi$,

$$
\max \left|A_{1} \frac{\bar{f}_{1}}{\bar{f}_{2}} \theta \sin \left(2 \pi \bar{f}_{1} t\right)\right| \ll \max \left|A_{2} \cos \left(\theta+\phi^{\prime}\right)\right|,
$$

the middle term of the argument of $F_{\text {ts }}$ in Eq. (5) can be ignored. This approximation removes the high-frequency wiggles of $\Delta \widetilde{f}_{2}(t)$ seen in Fig. 3, and we get a low pass filtered version of the frequency shift, $\Delta f_{2}(t)$ :

$$
\begin{aligned}
\Delta f_{2}(t)= & \frac{-\bar{f}_{2}}{2 \pi k_{2} A_{2}} \int_{-\pi}^{\pi} F_{\mathrm{ts}}\left(z_{1}(t)+A_{2} \cos \left(\theta+\phi^{\prime}\right)\right) \\
& \times \cos \left(\theta+\phi^{\prime}\right) d \theta
\end{aligned}
$$

We note that this frequency shift $\Delta f_{2}$ is significant only during the "contact time" $\left(T_{c}\right)$. This condition occurs around the negative peak of the first mode or when

$$
\frac{2 n+1}{2 f_{1}}-\frac{T_{c}}{2}<t<\frac{2 n+1}{2 f_{1}}+\frac{T_{c}}{2},
$$

where $n$ is an integer. If $T_{c}$ is small enough, for the values of $t$ given in Eq. (8) we have $\left|\sin \left(2 \pi \bar{f}_{1} t\right)\right|<2 \pi \bar{f}_{1} T_{c} / 2$, hence the requirement in Eq. (6) becomes

$$
\pi^{2} A_{1} \frac{\bar{f}_{1}^{2}}{\bar{f}_{2}} T_{c} \ll A_{2} .
$$

In this case, the frequency shift of the higher mode is given by an integration only over a single cycle of higher mode oscillations; therefore, in a sense, the vibration modes are decoupled.

Equation (7) is preferable over Eq. (3) since it generates a frequency shift curve without high-frequency wiggles and therefore without a phase ambiguity. Notice that the phase term $\phi^{\prime}$ in Eq. (7) can be dropped since $d\left(\Delta f_{2}(t)\right) / d \phi^{\prime}=0$, i.e., the low pass filtered frequency shift does not depend on the phase shift between the independent oscillators. Hence, we have

$$
\Delta f_{2}(t)=\frac{-\bar{f}_{2}}{2 \pi k_{2} A_{2}} \int_{-\pi}^{\pi} F_{\mathrm{ts}}\left(z_{1}(t)+A_{2} \cos \theta\right) \cos \theta d \theta .
$$

This is the frequency shift of the higher mode measured with a frequency demodulator of moderate bandwidth. $\Delta f_{2}(t)$ is also plotted in Fig. 3 for comparison.

Through integration by-parts where we take $d v=\cos \theta d \theta$ and $u=F_{\mathrm{ts}}\left(z_{1}(t)+A_{2} \cos \theta\right)$, a simpler, yet powerful expression of $\Delta f_{2}(t)$ is available:

$$
\begin{aligned}
\Delta f_{2}(t) \approx & -\frac{\bar{f}_{2}}{2 \pi k_{2}} \int_{-\pi}^{\pi} F_{\mathrm{ts}}^{\prime}\left(z_{1}(t)+A_{2} \cos \theta\right) \sin ^{2} \theta d \theta \\
= & -\frac{\bar{f}_{2}}{4 \pi k_{2}}\left[\int_{-\pi}^{\pi} F_{\mathrm{ts}}^{\prime}\left(z_{1}(t)+A_{2} \cos \theta\right) d \theta\right. \\
& \left.-\int_{-\pi}^{\pi} F_{\mathrm{ts}}^{\prime}\left(z_{1}(t)+A_{2} \cos \theta\right) \cos 2 \theta d \theta\right],
\end{aligned}
$$

where $F_{\mathrm{ts}}^{\prime}(\cdot)$ is the derivative of the tip-sample interaction force. If the third and higher order terms in the power series expansion of $F_{\mathrm{ts}}^{\prime}(\cdot)$ are negligible, i.e., as long as the condition

$$
A_{2} \ll 2\left|\frac{F_{\mathrm{ts}}^{\prime \prime}\left(z_{1}(t)\right)}{F_{\mathrm{ts}}^{\prime \prime \prime}\left(z_{1}(t)\right)}\right|
$$

is satisfied, we can write

$F_{\mathrm{ts}}^{\prime}\left(z_{1}(t)+A_{2} \cos \theta\right)=F_{\mathrm{ts}}^{\prime}\left(z_{1}(t)\right)+F_{\mathrm{ts}}^{\prime \prime}\left(z_{1}(t)\right) A_{2} \cos \theta$.

The second integral vanishes if Eq. (13) is substituted into Eq. (11), and we get a simpler result:

$$
\Delta f_{2}(t) \approx-\frac{\bar{f}_{2}}{4 \pi k_{2}} \int_{-\pi}^{\pi} F_{\mathrm{ts}}^{\prime}\left(z_{1}(t)+A_{2} \cos \theta\right) d \theta,
$$

with the combined necessary condition of

$$
\pi^{2} A_{1} \frac{\bar{f}_{1}^{2}}{\bar{f}_{2}} T_{c} \ll A_{2} \ll 2\left|\frac{F_{\mathrm{ts}}^{\prime \prime}\left(z_{1}(t)\right)}{F_{\mathrm{ts}}^{\prime \prime \prime}\left(z_{1}(t)\right)}\right| .
$$

Within this range of $A_{2}, \Delta f_{2}(t)$ is accurately described by the integral in Eq. (14). So, the higher mode vibration "samples" the gradient of the tip-sample force interaction and allows us to quantify $F_{\mathrm{ts}}(z)$ in a single cycle of the first mode vibrations $T_{1}$, while $\Delta f_{1}$ can be used to extract topographical features.

Suppose that the bandwidth of the frequency demodulator is not wide enough to capture the changes of $\Delta f_{2}(t)$ and an aggregate effect is observed. For example, a bandwidth smaller than $\bar{f}_{1}$ implies an averaging of Eq. (14) along $T_{1}$, and we get an averaged frequency shift, $\left\langle\Delta f_{2}\right\rangle$, of the higher mode vibrations

$$
\left\langle\Delta f_{2}\right\rangle \approx-\frac{\bar{f}_{2}}{4 \pi k_{2}} \int_{-\pi}^{\pi} F_{\mathrm{ts}}^{\prime}\left(Z_{0}+A_{1} \cos \phi\right) d \phi,
$$

which is the expression published recently by Kawai et al. ${ }^{15}$ to describe the observed frequency shift of the higher mode vibrations in bimodal dynamic force microscopy.

Equation (14) describes the frequency shift in terms of the force gradient. Solving the inverse problem, i.e., finding the force gradient from the measured frequency shift is more important. Starting from Eq. (14), we can derive an even simpler expression relating the force gradient to the measured frequency shift. If $A_{2}$ is sufficiently small, while still satisfying the left-hand side of Eq. (15), we can approximate $A_{2} \cos \theta$ with a square wave of the same peak values, and the integral in Eq. (14) can be simplified to give

$$
\begin{aligned}
\Delta f_{2}(t) & \approx-\frac{\bar{f}_{2}}{4 k_{2}}\left[F_{\mathrm{ts}}^{\prime}\left(z_{1}(t)+A_{2}\right)+F_{\mathrm{ts}}^{\prime}\left(z_{1}(t)-A_{2}\right)\right] \\
& \equiv-\frac{\bar{f}_{2}}{2 k_{2}} \overline{F_{\mathrm{ts}}^{\prime}\left(z_{1}(t)\right)},
\end{aligned}
$$

where the bar over the gradient function shows the average operation. Hence, the frequency shift is proportional to the average of two force gradient functions shifted by $2 A_{2}$ with respect to each other. On the other hand, in the limit where $A_{2}$ is very small, Eq. (17) reduces to

$$
F_{\mathrm{ts}}^{\prime}(z(t)) \approx-\frac{2 k_{2}}{\bar{f}_{2}} \Delta f_{2}(t),
$$

which shows that the tip-sample force gradient is directly proportional to the frequency shift. This equation is not very accurate, because of the condition in Eq. (15). It loses its validity when $A_{2}$ is made very small. It is better to use Eq. (17) and try to recover $F_{\mathrm{ts}}^{\prime}$ from it. 


\section{RECOVERY OF THE FORCE GRADIENT}

It is possible to recover $F_{\mathrm{ts}}^{\prime}$ from $\overline{F_{\mathrm{ts}}^{\prime}}$ by noting that

$$
\begin{gathered}
F_{\mathrm{ts}}^{\prime}\left(z-A_{2}\right)=2 \overline{F_{\mathrm{ts}}^{\prime}(z)}-F_{\mathrm{ts}}^{\prime}\left(z+A_{2}\right), \\
F_{\mathrm{ts}}^{\prime}(z) \rightarrow 0 \text { for } z \rightarrow \infty .
\end{gathered}
$$

For this purpose, first an interpolation is necessary to get equally spaced samples of $F_{\mathrm{ts}}^{\prime}$ in $z$ from equally spaced samples in $t$. The first recovery algorithm can be written as follows:

1. From the measured $\Delta \widetilde{f}_{2}(t)$ for $t_{i}=t_{i-1}+\Delta t$, determine $\overline{F_{\mathrm{ts}}^{\prime}\left(z\left(t_{i}\right)\right)}$ using Eq. (17).

2. Interpolate $\overline{F_{\mathrm{ts}}^{\prime}\left(z\left(t_{i}\right)\right)}$ to get $\overline{F_{\mathrm{ts}}^{\prime}\left(z_{(j)}\right)}$ with $z_{(j+1)}=z_{(j)}-$ $A_{2} / m$, where $m$ is an integer chosen to give a sufficient sampling distance in $z . j$ is the sample index. $z_{(0)}$ is chosen sufficiently large so that $F_{\mathrm{ts}}^{\prime}\left(z_{(0)}\right)=0$.

3. Use $F_{\mathrm{ts}}^{\prime}\left(z_{(j+m)}\right)=2 \overline{F_{\mathrm{ts}}^{\prime}\left(z_{(j)}\right)}-F_{\mathrm{ts}}^{\prime}\left(z_{(j-m)}\right)$ for $j=0,1$, $2, \ldots$ to recover $F_{\mathrm{ts}}^{\prime}$ function at equally spaced intervals. For initialization we choose $F_{\mathrm{ts}}^{\prime}\left(z_{(j)}\right)=0$ for $j<0$.

This algorithm tries to recover the force gradient from the average value starting from very large $z$ values where the gradient is known to vanish and work its way in an iterative manner toward lower $z$ values. Since the algorithm is sufficiently simple, it can be implemented in real time while the data points are being captured. If the noise between the samples are uncorrelated, the recovery algorithm degrades the signal-to-noise ratio by about $10 \log _{10} 5=7 \mathrm{~dB}$. This is a significant loss in signal quality.

One can obtain a better performance in recovery using a more computationally intensive and hence possibly an off-line method. For the second algorithm, assume a model for $F_{\mathrm{ts}}(z)$ and find the parameters of the model to satisfy Eq. (3) in the least-squares sense using an optimization method. A possible model is given by ${ }^{11}$

$$
F_{\mathrm{ts}}(z)= \begin{cases}-F_{\max } /\left[1+30\left(z-a_{0}\right)^{2}\right] & \text { for } z \geqslant a_{0}, \\ -F_{\max }+S_{\text {rep }}\left(z-a_{0}\right)^{2} & \text { for } z<a_{0},\end{cases}
$$

where $F_{\max }$ represents the maximum of the attractive forces, $S_{\text {rep }}$ is the strength of the repulsive interaction, and $a_{0}$ is the interatomic distance separating attractive and repulsive force regimes. All forces are in $\mathrm{nN}$, all distances are $\mathrm{nm}$, and $S_{\text {rep }}$ has units of $\mathrm{nN} \mathrm{nm}^{-2}$.

\section{SIMULATIONS}

We test the validity of Eq. (14) using a time-domain electrical circuit simulator, SPICE. We treat the vibration modes of the cantilever using two series RLC circuits, therefore assuming a point mass model. Other vibration modalities are simply ignored. Two positive feedback loops are included to maintain $90^{\circ}$ of phase shift between the vibrations of the tip and the actuation. RMS detectors for each vibration mode followed by corresponding proportional-integral controllers maintain constant amplitude vibrations of the modes regardless of the strength of the interaction and hence regardless of the base-sample distance. The vibration modes are coupled through a nonlinear circuit component, output of which is described by Eq. (21). This equation is preferred over the widely used Derjaguin, Muller, and Toporov model ${ }^{16}$ of the tip-sample forces, since the derivative of $F_{\mathrm{ts}}$ in Eq. (21)

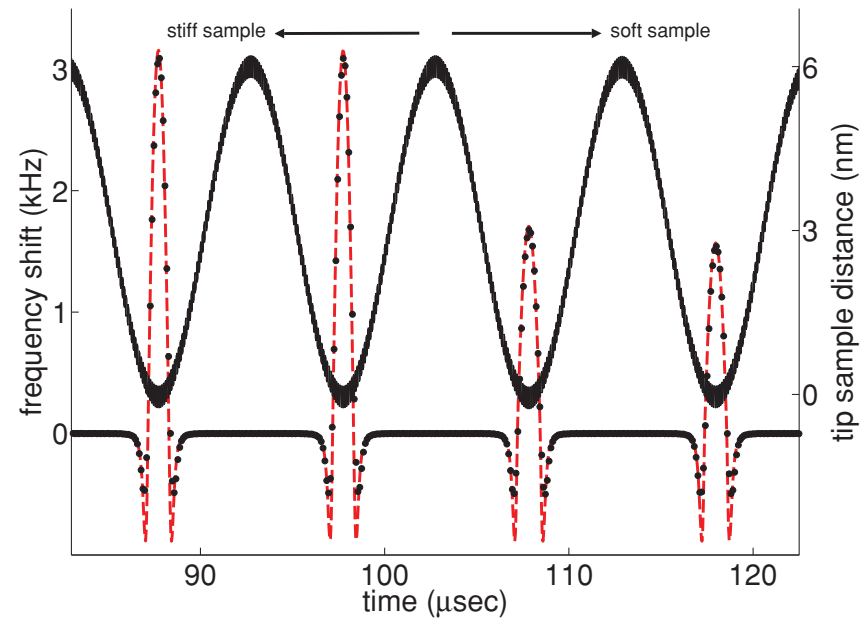

FIG. 4. (Color online) Frequency shift of the higher mode (dots) and the tip-sample distance (solid line) with respect to time. The dots are obtained from the numerical simulation and the dashed line is calculated using Eq. (18) and assuming that $F_{\text {ts }}$ is given by Eq. (21). $A_{1}=3 \mathrm{~nm}, A_{2}=0.2 \mathrm{~nm}, Z_{0}=3 \mathrm{~nm}, \bar{f}_{1}=100 \mathrm{kHz}, \bar{f}_{2} \approx 100 \bar{f}_{1}$. $F_{\text {max }}=5 \mathrm{nN}, S_{\text {rep }}=150 \mathrm{nN} \mathrm{nm}^{-2}$ for $t<100 \mu \mathrm{s}$ and $S_{\text {rep }}=$ $75 \mathrm{nN} \mathrm{nm}^{-2}$ for $t>100 \mu \mathrm{s}$. Measurement bandwidth is $20 \bar{f}_{1}$.

with respect to tip-sample distance is continuous regardless of the parameters used. For all simulations, $\bar{f}_{1}=100 \mathrm{kHz}$, first mode stiffness $k_{1}=10 \mathrm{~N} / \mathrm{m}, Q_{1}=200$, and $Q_{2}=500$. The resonant frequency of the higher mode $\left(\bar{f}_{2}\right)$ is either 3 or $10 \mathrm{MHz}$. Stiffness of the higher mode is given by $k_{2}=k_{1}\left(\bar{f}_{2} / \bar{f}_{1}\right)^{2}$.

Figure 4 shows the simulation results for the instantaneous frequency shift of the higher mode as a function of time. The sample is assumed to be perfectly flat, but it has two regions with different force curves. As seen in the figure, the frequency shifts when the tip is nearest to the sample. The scan speed is limited by the period of the low-frequency drive. As expected, the sensitivity of the higher mode vibrations

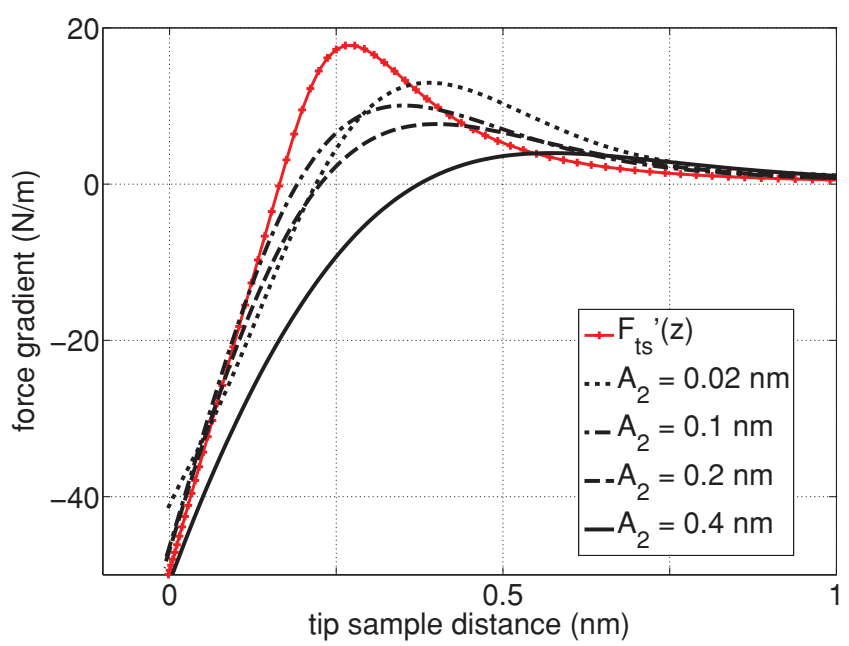

FIG. 5. (Color online) The actual force gradient $F_{\mathrm{ts}}^{\prime}(z)$ and the force gradient curves obtained from the frequency shift data using Eq. (18) with respect to tip-sample distance. $A_{1}=10 \mathrm{~nm}$, $Z_{0}=10 \mathrm{~nm}, \bar{f}_{1}=100 \mathrm{kHz}, \bar{f}_{2} \approx 100 \bar{f}_{1}, F_{\max }=5 \mathrm{nN}$, and $S_{\text {rep }}=$ $150 \mathrm{nN} \mathrm{nm}^{-2}$, where $F_{\mathrm{ts}}$ is given by Eq. (21). 


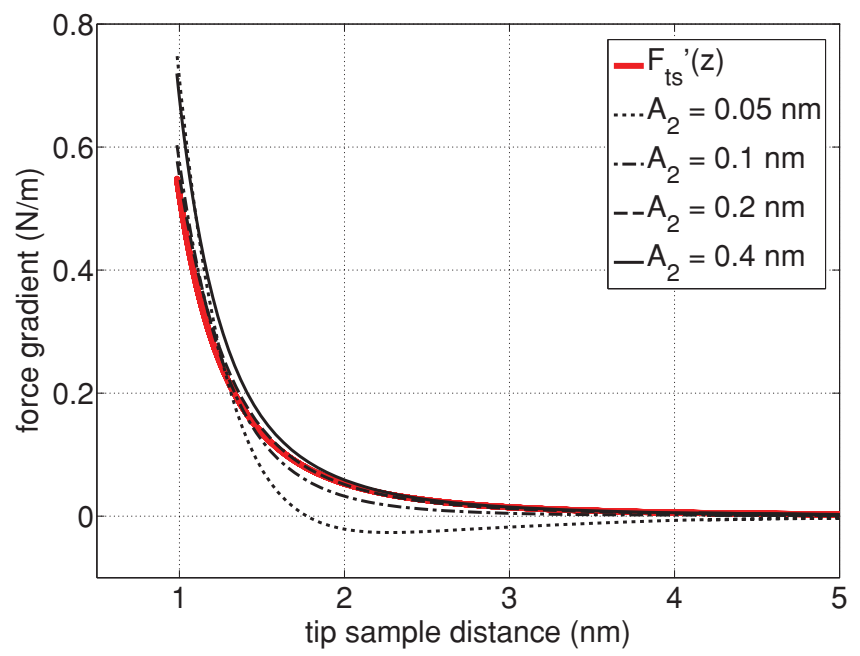

FIG. 6. (Color online) Actual force gradient $F_{\mathrm{ts}}^{\prime}(z)$ and the force gradient curves obtained from the frequency shift data using Eq. (18) with respect to tip-sample distance. $A_{1}=3 \mathrm{~nm}, Z_{0}=4 \mathrm{~nm}, \bar{f}_{1}=$ $100 \mathrm{kHz}, \bar{f}_{2} \approx 30 \bar{f}_{1}$, and $F_{\max }=5 \mathrm{nN}$, where $F_{\text {ts }}$ is given by Eq. (21).

to surface property variations are instantaneous, therefore independent of the quality factor of the higher mode.

The force gradient can be calculated from the frequency shift data, assuming that Eq. (18) is sufficient to describe the dynamics. Figure 5 shows such a calculation along with the actual curve for $F_{\mathrm{ts}}^{\prime}(z)$. The accuracy of the force gradient degrades as $A_{2}$ is increased. This is expected, since the right-hand condition in Eq. (15) is violated. On the other extreme, when $A_{2}$ becomes too small, the accuracy also begins to deteriorate. In this case, the left-hand condition of Eq. (15) is violated. The effect is more pronounced in Fig. 6, where $\bar{f}_{2}=3 \mathrm{MHz}$, and base-sample separation $Z_{0}=4 \mathrm{~nm}$. In this case, tip oscillates only in the attractive regime. Reconstruction is almost perfect for $A_{2}=0.2 \mathrm{~nm}$ for which Eq. (15) holds. However, for $A_{2}=0.1-0.05 \mathrm{~nm}$, the left-hand condition of Eq. (15) is not satisfied, hence the distortion in the gradient reconstruction.

Notice that in Fig. 5 the discrepancy between the actual force gradient and the force gradient calculated from the frequency shift data using Eq. (18) is maximum for the region where the force gradient is maximum. This is the transition region between the attractive and repulsive forces for which the right-hand side of Eq. (15) is violated regardless of the chosen higher mode amplitude. If the characteristics of the

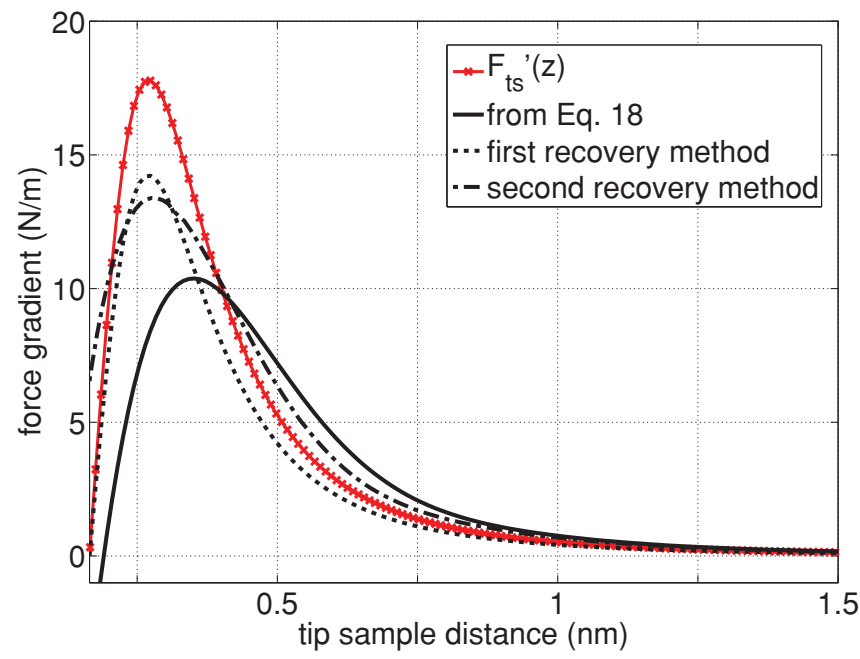

FIG. 7. (Color online) Actual force gradient $F_{\mathrm{ts}}^{\prime}(z)$ and the force gradient estimate obtained using Eq. (18) along with the force gradient curves reconstructed using the first recovery algorithm and the second algorithm. $A_{1}=10 \mathrm{~nm}, A_{2}=0.1 \mathrm{~nm}, Z_{0}=10 \mathrm{~nm}, \bar{f}_{1}=100 \mathrm{kHz}$, $\bar{f}_{2} \approx 100 \bar{f}_{1}, F_{\max }=5 \mathrm{nN}$, and $S_{\text {rep }}=150 \mathrm{nN} \mathrm{nm}^{-2}$, where $F_{\text {ts }}$ is given by Eq. (21).

force curve within this transition region is of interest, it is worthwhile to use one of the recovery algorithms. Figure 7 shows the results of the recovery algorithms in comparison to uncorrected data. The recovered force gradients within the transition region are not perfect, but they are definitely better than the uncorrected version.

\section{CONCLUSION}

We derived expressions describing the time-dependent frequency shift of the higher mode vibrations which is related to an averaging over the force gradient of the tip-sample force interaction. We have shown that frequency shifts of the higher mode can be used to extract tip-sample forces. We proposed two methods of improving the accuracy of reconstruction from the measured frequency shift data.

Finally, we note that bimodal frequency modulation AFM is a strong candidate for force spectroscopy especially in a vacuum environment where the large quality factor of the vibration mode limits the imaging bandwidth of amplitude modulation techniques, namely, harmonic imaging and bimodal amplitude modulation AFM. Using the proposed scheme would allow a high-quality quantification of the mechanical properties of the sample without any degradation of the scan speed.

\footnotetext{
*aksoy@ee.bilkent.edu.tr

†atalar@ee.bilkent.edu.tr

${ }^{1}$ G. Binnig, C. F. Quate, and C. Gerber, Phys. Rev. Lett. 56, 930 (1986).

${ }^{2}$ H. Holscher, W. Allers, U. D. Schwarz, A. Schwarz, and R. Wiesendanger, Phys. Rev. Lett. 83, 4780 (1999).

${ }^{3}$ F. Giessibl, Appl. Phys. Lett. 78, 123 (2001).
}

${ }^{4}$ M. Lantz, H. Hug, R. Hoffmann, P. van Schendel, P. Kappenberger, S. Martin, A. Baratoff, and H. Guntherodt, Science 291, 2580 (2001).

${ }^{5}$ F. Giessibl, Rev. Mod. Phys. 75, 949 (2003).

${ }^{6}$ O. Sahin, C. F. Quate, O. Solgaard, and A. Atalar, Phys. Rev. B 69, 165416 (2004).

${ }^{7}$ O. Sahin, S. Magonov, C. Su, C. F. Quate, and O. Solgaard, Nat. Nanotechnol. 2, 507 (2007). 
${ }^{8}$ N. F. Martinez, S. Patil, J. R. Lozano, and R. Garcia, Appl. Phys. Lett. 89, 153115 (2006).

${ }^{9}$ S. Patil, N. F. Martinez, J. R. Lozano, and R. Garcia, J. Mol. Recognit. 20, 516 (2007).

${ }^{10}$ J. R. Lozano and R. Garcia, Phys. Rev. B 79, 014110 (2009).

${ }^{11}$ G. Chawla and S. D. Solares, Meas. Sci. Technol. 20, 015501 (2009).

${ }^{12}$ J. Kokavecz, O. Marti, P. Heszler, and A. Mechler, Phys. Rev. B 73, 155403 (2006).
${ }^{13}$ T. Albrecht, P. Grutter, D. Horne, and D. Rugar, J. Appl. Phys. 69, 668 (1991).

${ }^{14}$ Y. Mitani, M. Kubo, K.-i. Muramoto, and T. Fukuma, Rev. Sci. Instrum. 80, 083705 (2009).

${ }^{15}$ S. Kawai, T. Glatzel, S. Koch, B. Such, A. Baratoff, and E. Meyer, Phys. Rev. Lett. 103, 220801 (2009).

${ }^{16}$ B. Derjaguin, V. Muller, and Y. Toporov, J. Colloid Interface Sci. 53, 314 (1975). 